\title{
Optimizing Collegiate EMS Resources During Major Events
}

\author{
Katherine R. Luchette*, AEMT-Cardiac; Oliver G. Isik*, EMT; Heather Rybasack-Smith, MD, \\ MPH; Nicholas Asselin, DO, MS; Thomas J. Martin, AEMT-Cardiac
}

*Contributed equally to this work.

\section{POSTER PRESENTATION ABSTRACT}

Introduction: Each year, Brown University holds Spring Weekend (SWE), an outdoor concert drawing 6,000 attendees per day. Mass gatherings are prone to developing into mass casualty incidents (MCIs) due to unplanned events and attacks. Though campus venues face comparable risks, collegiate resources are more commonly and predictably overwhelmed by medical surge from trauma and/or toxicological emergencies. These 'planned MCIs' offer unique training opportunities and inform disaster planning. Brown EMS (BEMS) provides primary medical coverage for SWE and develops annual response plans using data-driven evaluation. Program Development $\&$ Implementation: BEMS establishes EMS Operations during major events using a modified ICS structure. Whereas our ALS/BLS-licensed ambulance and non-transport SUV are routinely in-service, venue resources are augmented by dedicated apparatuses and pairs of EMTs assigned to ground details. By increasing its resources, BEMS aims to increase venue capacity, optimize response, and limit mutual-aid requirements. The number and proportion of transports by external agencies consistently increased over 2014-2016: 25.0\% (3/12), 31.3\% (5/16), and 45.0\% (9/20) during the six-hour Friday concert, respectively. For SWE 2017, we hypothesized that renting and staffing an additional ALS ambulance with BEMS personnel would decrease mutual-aid utilization. Program Evaluation: To control for annual variations, we compared mutual-aid utilization in 2017 against aggregate utilization during the three prior years and observed no significant difference: $20.8 \%(5 / 24)$ vs. $35.4 \%(17 / 48)(\mathrm{p}=0.21)$. This suggests that in response planning, the temporal density of patient presentations during surge conditions may better predict mutual-aid requirements than historical call volume. Discussion/Conclusion: Though mutual-aid is a cornerstone of MCI management, collegiate systems may face unique pressures to decrease its utilization if students are exposed to additional billing. Expanding our transport sector in 2017 did not significantly decrease the proportion of patients requiring mutual-aid transport. To optimize incident response, collegiate services may instead consider establishing on-site triage/treatment areas and concurrently transporting low-acuity patients.

Author Affiliations: From Emergency Medical Services, Brown University, Providence, RI, USA (O.G.I, K.R.L., T.J.M.); Division of Emergency Medical Services, Department of Emergency Medicine, The Warren Alpert Medical School of Brown University, Providence, RI, USA (H.R., N.A.); Program in Emergency Digital Health Innovation, Department of Emergency Medicine, The Warren Alpert Medical School of Brown University, Providence, RI, USA (T.J.M.).

Address for Correspondence: Thomas J. Martin, AEMT-Cardiac, The Warren Alpert Medical School of Brown University, 69 Brown St., Box 2784, Providence, RI, USA. E-mail: thomas_martin@brown.edu

Conflicts of Interest/Funding Sources: By the JCEMS Submission Declaration Form, all authors are required to disclose all potential conflicts of interest and funding sources. The authors declared that they have no conflicts of interest. The authors declared that they did not receive funding to conduct the program or research associated with this work.

Ethical Compliance: The authors attest that the research associated with this abstract was conducted in accordance with the JCEMS Ethics Guidelines.

Submission History: Received January 14, 2018; accepted for presentation and publication February 21, 2018.

Poster Presentation: This abstract was presented as a poster at the Academic Poster Session of the $25^{\text {th }}$ Annual Conference of the National Collegiate Emergency Medical Services Foundation; February 24, 2018; Philadelphia, PA, USA. The poster is available for download on the JCEMS website.

Published Online: May 13, 2018

Published in Print: August 13, 2018 (Volume 1: Supplemental 1)

Reviewer Information: In accordance with JCEMS editorial policy, poster presentation abstracts undergo double-blind peer-review by at least two reviewers (JCEMS Editorial Board members and/or independent reviewers) prior to acceptance for presentation and publication. JCEMS thanks the anonymous reviewers who contributed to the review of this work.

Copyright: (C) 2018 Isik, Luchette, Rybasack-Smith, Asselin, \& Martin. This is an open access abstract distributed under the terms of the Creative Commons Attribution 4.0 International (CC BY 4.0) License, which permits unrestricted use, distribution, and reproduction in any medium, provided the original author and source are credited. The full license is available at: https://creativecommons.org/licenses/by/4.0/

Electronic Link: https://doi.org/10.30542/JCEMS.2018.01.S1.09 